\title{
215. Integration of Equations of Imschenetsky Type by Integrable Systems
}

\author{
By Michihiko Matsuda \\ Department of Mathematics, Osaka University \\ (Comm. by Kunihiko KodaIra, M. J. A., Sept. 13, 1971)
}

1. Introduction. Generalizing the method of integration due to Monge, the author gave a method of integration by integrable systems in [1] and [2]. Here we shall prove the following

Theorem. Transform an equation of Imschenetsky type by one of the associated Imschenetsky transformations. Then the transformed equation is solved by integrable systems of order $n-1$, if and only if the original equation is solved by integrable systems of order $n$.

This is a generalization of results in [1], [2] obtained for the Laplace transformation associated with a linear hyperbolic equation, and for the Imschenetsky transformation associated with an equation of Laplace type. In the second case the theorem was proved only for $n=1,2$. In both the cases the author obtained the invariants of the equation whose vanishing is a necessary and sufficient condition in order that the equation may be solved by integrable systems of order $n$, and proved that the invariants for the original equation to be solved by integrable systems of order $n$ are transformed to those for the transformed equation to be solved by integrable systems of order $n-1$. Here we shall prove the theorem directly, without obtaining the invariants of the equations.

2. Integrable systems of order $n$. Let us try to solve the Cauchy problem of an equation of type

$$
s+f(x, y, z, p, q)=0,
$$

integrating ordinary differential equations, in the space of $\left(x, y, z, p, q_{1}\right.$, $\left.\cdots, q_{n}\right)$. Here, $p=\partial z / \partial x, q=\partial z / \partial y, \quad s=\partial^{2} z / \partial x \partial y$, and $q_{i}=\partial^{i} z / \partial y^{i}$ $\left(q_{1}=q\right)$. The Cauchy problem in this space involving the derivatives of higher order is to find a two-dimensional submanifold which satisfies

$$
\begin{aligned}
d z-p d x-q d y & =d q_{1}+f_{0} d x-q_{2} d y=d q_{2}+f_{1} d x-q_{3} d y \\
& =\cdots=d q_{n-1}+f_{n-2} d x-q_{n} d y=0,
\end{aligned}
$$

and contains a given initial curve satisfying (2). Here, $f_{i}$ is a function of $\left(x, y, z, p, q_{1}, \cdots, q_{i+1}\right)$ defined inductively by

with

$$
f_{i}=\left(G_{i}-f \frac{\partial}{\partial p}\right) f_{i-1} \quad(i \geqq 1), \quad f_{0}=f
$$




$$
G_{i}=\frac{d}{d y}+\sum_{j=1}^{i} q_{j+1} \frac{\partial}{\partial q_{j}} \quad(i \geqq 1), \quad \frac{d}{d y}=\frac{\partial}{\partial y}+q \frac{\partial}{\partial z} .
$$

A system of ordinary differential equations

$$
\frac{d x}{0}=\frac{d y}{1}=\frac{d z}{q_{1}}=\frac{d p}{-f}=\frac{d q_{1}}{q_{2}}=\cdots=\frac{d q_{n-1}}{q_{n}}=\frac{d q_{n}}{u}
$$

with a function $u$ of $\left(x, y, z, p, q_{1}, \cdots, q_{n}\right)$ is called an integrable system of order $n$, if $u$ is a solution of the following system of two equations

$$
\frac{\partial u}{\partial p}=0, \quad\left(\frac{d}{d x}-\sum_{i=1}^{n} f_{i-1} \frac{\partial}{\partial q_{i}}\right) u+\frac{\partial f}{\partial q} u+\left(G_{n-1}-f \frac{\partial}{\partial p}\right) f_{n-1}=0,
$$

where $d / d x=\partial / \partial x+p \partial / \partial z$. Suppose that along the initial curve satisfying (2) we have $d q_{n}+f_{n-1} d x-u d y=0$. Then the surface obtained by integrating (3) under the given initial condition satisfies (2) and $d q_{n}$ $+f_{n-1} d x-u d y=0$, if the system (3) is integrable. Hence, in this case, the surface thus obtained gives a solution of the Cauchy problem.

3. Integrability condition. Any system of linear partial differential equations of the first order with one unknown function can be prolonged either to a complete system or to an incompatible system by adding compatibility conditions. If we get a complete system consisting of $r$ independent equations by this prolongation, the original system is said to have the rank $m-r$, where $m$ is the number of the independent variables. The equation (1) is said to be solved by integrable systems of order $n$, if the system (4) has the rank greater than zero. In this case the Cauchy problem is solved by integrating an integrable system of order $n$ for any initial curve satisfying (2), since we can find such a solution $u$ of (4) that satisfies $d q_{n}+f_{n-1} d x-u d y=0$ along the given initial curve.

Suppose that the equation (1) is linear with respect to $p$;

$$
s+M(x, y, z, q) p+N(x, y, z, q)=0 .
$$

Then the second equation of (4) is written in the form

where

$$
p\left(Z_{n} u+\frac{\partial M}{\partial q} u+M_{n}\right)+X_{n} u+\frac{\partial N}{\partial q} u+N_{n}=0,
$$

$$
\begin{array}{ll}
Z_{n}=\frac{\partial}{\partial z}-\sum_{i=1}^{n} A_{i-1} \frac{\partial}{\partial q_{i}}, & M_{n}=G_{n-1} A_{n-1}-M A_{n-1}, \\
X_{n}=\frac{\partial}{\partial x}-\sum_{i=1}^{n} B_{i-1} \frac{\partial}{\partial q_{i}}, & N_{n}=G_{n-1} B_{n-1}-N A_{n-1},
\end{array}
$$

and $A_{i}, B_{i}(i \geqq 0)$ are defined inductively by

$$
A_{i}=G_{i} A_{i-1}-M A_{i-1}, \quad B_{i}=G_{i} B_{i-1}-N A_{i-1}, \quad A_{0}=M, \quad B_{0}=N .
$$

The functions $M_{n}, N_{n}, A_{i}, B_{i}(i \geqq 0)$ are independent of $p$. Hence, the equation (5) is solved by integrable systems of order $n$, if and only if the system of two equations 


$$
Z_{n} u+\frac{\partial M}{\partial q} u+M_{n}=0, \quad X_{n} u+\frac{\partial N}{\partial q} u+N_{n}=0
$$

with independent variables $\left(x, y, z, q_{1}, \cdots, q_{n}\right)$ has the rank greater than zero.

4. Imschenetsky transformation. A set of four relations

$$
x^{\prime}=x, \quad y^{\prime}=y, \quad z^{\prime}=h(x, y, z, q), \quad p^{\prime}=k(x, y, z, q)
$$

between $\left(x, y, z, p, q ; x^{\prime}, y^{\prime}, z^{\prime}, p^{\prime}, q^{\prime}\right)$ is called an Imschenetsky transformation, if it satisfies

$$
\frac{\partial h}{\partial q} \neq 0, \quad \frac{\partial(h, k)}{\partial(z, q)} \neq 0 .
$$

It gives the following transformation between the two equations from

$$
\frac{\partial h}{\partial q} s+\frac{\partial h}{\partial z} p+\frac{\partial h}{\partial x}-k=0
$$

to

$$
\frac{\partial h}{\partial q} s^{\prime}-\frac{\partial k}{\partial q} q^{\prime}-\frac{d k}{d y} \frac{\partial h}{\partial q}+\frac{d h}{d y} \frac{\partial k}{\partial q}=0,
$$

where we replace $x, y, z, q$ in the second equation by

$$
x=x^{\prime}, \quad y=y^{\prime}, \quad z=h^{\prime}\left(x^{\prime}, y^{\prime}, z^{\prime}, p^{\prime}\right), \quad q=k^{\prime}\left(x^{\prime}, y^{\prime}, z^{\prime}, p^{\prime}\right),
$$

solving (7) with respect to $x, y, z, q$. Take a solution $z=\phi(x, y)$ of (8). Then the surface $z^{\prime}=h\left(x^{\prime}, y^{\prime}, \phi\left(x^{\prime}, y^{\prime}\right), \phi_{y}\left(x^{\prime}, y^{\prime}\right)\right)$ gives a solution of (9). Conversely take a solution $z^{\prime}=\phi^{\prime}\left(x^{\prime}, y^{\prime}\right)$ of (9). Then the surface $z$ $=h^{\prime}\left(x, y, \phi^{\prime}(x, y), \phi_{x^{\prime}}^{\prime}(x, y)\right)$ gives a solution of (8). These two transformations are the inverse of each other.

The original equation (8) is linear with respect to $p$. An equation of type (5) can be the original equation of an Imschenetsky transformation, if and only if its first invariant

$$
X_{1} M-Z_{1} N=\frac{\partial M}{\partial x}-N \frac{\partial M}{\partial q}-\frac{\partial N}{\partial z}+M \frac{\partial N}{\partial q}
$$

does not vanish. In this case the equation (5) is called an equation of Imschenetsky type.

5. Proof of Theorem. Suppose that an equation of Imschenetsky type (5) is transformed to an equation

$$
s^{\prime}+f^{\prime}\left(x^{\prime}, y^{\prime}, z^{\prime}, p^{\prime}, q^{\prime}\right)=0
$$

by the Imschenetsky transformation (7). Then we have

$$
Z_{1} h=0, \quad X_{1} h=k, \quad-f^{\prime}=\frac{d k}{d y}+\left(\frac{\partial h}{\partial q}\right)^{-1}\left(q^{\prime}-\frac{d h}{d y}\right) \frac{\partial k}{\partial q} .
$$

Let us transform the independent variables $\left(x, y, z, q_{1}, \cdots, q_{n}\right)$ and the unknown function $u$ of the system (6) to $\left(x^{\prime}, y^{\prime}, z^{\prime}, p^{\prime}, q_{1}^{\prime}, \cdots, q_{n-1}^{\prime}\right)$ and $u^{\prime}$ respectively by

$$
x^{\prime}=x, \quad y^{\prime}=y, \quad z^{\prime}=h, \quad p^{\prime}=k, \quad q^{\prime}=G_{1} h=\frac{d h}{d y}+\frac{\partial h}{\partial q} q_{2},
$$




$$
q_{i}^{\prime}=G_{i} G_{i-1} \cdots G_{1} h=G_{i-1}\left(G_{i-1} \cdots G_{1} h\right)+\frac{\partial h}{\partial q} q_{i+1}, \quad 1<i<n
$$

and

$$
u^{\prime}=G_{n-1}\left(G_{n-1} \cdots G_{1} h\right)+\frac{\partial h}{\partial q} u .
$$

Then the first and the second equations of (6) are expressed in the forms

$$
-\left(\frac{\partial h}{\partial q}\right)^{-2} \frac{\partial(h, k)}{\partial(z, q)} \frac{\partial u^{\prime}}{\partial p^{\prime}}=0
$$

and

$$
\begin{aligned}
&\left(\frac{\partial h}{\partial q}\right)^{-1}\left\{\frac{d u^{\prime}}{\partial x^{\prime}}-\sum_{i=1}^{n-1} f_{i-1}^{\prime} \frac{\partial u^{\prime}}{\partial q_{i}^{\prime}}+\frac{\partial f^{\prime}}{\partial q^{\prime}} u^{\prime}+\left(G_{n-2}^{\prime}-f^{\prime} \frac{\partial}{\partial p^{\prime}}\right) f_{n-2}^{\prime}\right\} \\
&+\left(\frac{\partial h}{\partial q}\right)^{-1} X_{1} k \frac{\partial u^{\prime}}{\partial p^{\prime}}=0
\end{aligned}
$$

respectively, where

$$
G_{i}^{\prime}=\frac{d}{d y^{\prime}}+\sum_{j=1}^{i} q_{j+1}^{\prime} \frac{\partial}{\partial q_{j}^{\prime}}, \quad f_{i}^{\prime}=\left(G_{i}^{\prime}-f^{\prime} \frac{\partial}{\partial p^{\prime}}\right) f_{i-1}^{\prime}, \quad f_{0}^{\prime}=f^{\prime} .
$$

It can be proved by

$$
\begin{gathered}
Z_{n} z^{\prime}=Z_{n} h=0, \quad X_{n} z^{\prime}=X_{n} h=k, \quad Z_{n} p^{\prime}=Z_{n} k=-\left(\frac{\partial h}{\partial q}\right)^{-1} \frac{\partial(h, k)}{\partial(z, q)}, \\
Z_{n} q_{i}^{\prime}=Z_{n}\left(G_{i} \cdots G_{1}\right) h=0, \quad X_{n} q_{i}^{\prime}=X_{n}\left(G_{i} \cdots G_{1}\right) h=-f_{i-1}^{\prime}, \quad 1 \leqq i<n,
\end{gathered}
$$
and

$$
\begin{gathered}
Z_{n}\left(\frac{\partial h}{\partial q}\right)^{-1}=-\frac{\partial M}{\partial q}\left(\frac{\partial h}{\partial q}\right)^{-1}, \quad X_{n}\left(\frac{\partial h}{\partial q}\right)^{-1}=-\left(\frac{\partial N}{\partial q}-\frac{\partial f^{\prime}}{\partial q^{\prime}}\right)\left(\frac{\partial h}{\partial q}\right)^{-1}, \\
Z_{n} G_{n-1}\left(G_{n-1} \cdots G_{1}\right) h=M_{n} \frac{\partial h}{\partial q} \\
X_{n} G_{n-1}\left(G_{n-1} \cdots G_{1}\right) h= \\
=N_{n} \frac{\partial h}{\partial q}-\left(G_{n-2}^{\prime}-f^{\prime} \frac{\partial}{\partial p^{\prime}}\right) f_{n-2}^{\prime} \\
-\frac{\partial f^{\prime}}{\partial q^{\prime}} G_{n-1}\left(G_{n-1} \cdots G_{1}\right) h
\end{gathered}
$$

These identities can be obtained by

$$
\begin{gathered}
{\left[Z_{i+1}, G_{i}\right]=-M Z_{i}+G_{i} A_{i} \frac{\partial}{\partial q_{i+1}}, \quad i \geqq 1,} \\
{\left[X_{i+1}, G_{i}\right]=-N Z_{i}+G_{i} B_{i} \frac{\partial}{\partial q_{i+1}}, \quad i \geqq 1,} \\
G_{i}=G_{i-1}^{\prime}-f^{\prime} \frac{\partial}{\partial p^{\prime}}+\sum_{j=i}^{n-1} G_{i}\left(G_{j} \cdots G_{1}\right) h \frac{\partial}{\partial q_{j}^{\prime}}, \quad i \geqq 1 \quad\left(G_{0}^{\prime}=\frac{d}{d y^{\prime}}\right),
\end{gathered}
$$

where$$
Z_{i}=\frac{\partial}{\partial z}-\sum_{j=1}^{i} A_{j-1} \frac{\partial}{\partial q_{j}}, \quad X_{i}=\frac{\partial}{\partial x}-\sum_{j=1}^{i} B_{j-1} \frac{\partial}{\partial q_{j}}, \quad i \geqq 1 .
$$

Hence, the system (6) with independent variables $\left(x, y, z, q_{1}, \cdots, q_{n}\right)$ and the system of two equations 


$$
\frac{\partial u^{\prime}}{\partial p^{\prime}}=\frac{d u^{\prime}}{d x^{\prime}}-\sum_{i=1}^{n-1} f_{i-1}^{\prime} \frac{\partial u^{\prime}}{\partial q_{i}^{\prime}}+\frac{\partial f^{\prime}}{\partial q^{\prime}} u^{\prime}+\left(G_{n-2}^{\prime}-f^{\prime} \frac{\partial}{\partial p^{\prime}}\right) f_{n-2}^{\prime}=0
$$

with independent variables $\left(x^{\prime}, y^{\prime}, z^{\prime}, p^{\prime}, q_{1}^{\prime}, \cdots, q_{n-1}^{\prime}\right)$ have the same rank.

\section{References}

[1] M. Matsuda: Two methods of integrating Monge-Ampère's equations. Trans. Amer. Math. Soc., 150, 327-343 (1970).

[2] - : Two methods of integrating Monge-Ampère's equations. II (to appear in Trans. Amer. Math. Soc., 167 (1972)). 